\section{Subtlety and Change}

There are some strange changes under way in our world. We constantly hear the refrain of the massive chaos around us, yet the allure of such a large, looming flux may distract us from something more important: the countless tiny, nuanced, and fundamental ways in which our culture and society are advancing. This issue of interactions describes these subtleties and teases them out of the greater topics that we've grown accustomed to discussing: environmental change, the role of education and government in a technological society, and the nature of behavior.

Jon Innes describes the idea of cultural acceptance as a theoretical requirement for exploring the coming future, and the need to better identify trends in the world around us. This relates to Don Norman's call for "translational developers" - those who are able to take research findings and articulate directional actions. And Katie Minardo Scott describes the challenge of synthesis - the relationship between a designer and the data that can be so overwhelming.

A similar investigative depth is found in the piece by Carl DiSalvo, Phoebe Sengers, and Hrönn Brynjarsdóttir, who explore the manner in which sustainability has impacted HCI and academic research. No longer a simple colloquialism of "reduce, reuse, and recycle," sustainability research and design now require an acknowledgment of the political differences involved in the discipline. This demands a more nuanced discus- sion of topics, as well as a more direct connection between research and practice.

The same political connotations are present in a conversation of education reform. Cover story author Dennis Littky - the co-founder and co-director of Big Picture Learning-describes the need for fundamental shifts in high school education in the U.S. As he ponders why dropout rates average 50 percent, he describes a new model for learning, one that rejects traditions and begs a clearer understanding of the culture of our teenagers.

This issue brings these topics, along with Liz Danzico's discussion of the subtleties of timing in design and conversation, Fred Scharmen's thoughts on the intricacies of the social Web, and Nicholas Carr's reflections on the state of our technological culture. The topics are broad but the analysis is nuanced and sharp: The changes to our world can be unpacked from their unwieldy topical containers so we can analyze, discuss, and embrace the shifts taking place.

We hope you find value in these perspectives and can integrate them into your daily work-the work that is supporting these changes and helping drive them to fruition.

一Jon Kolko eic@interactions.acm.org

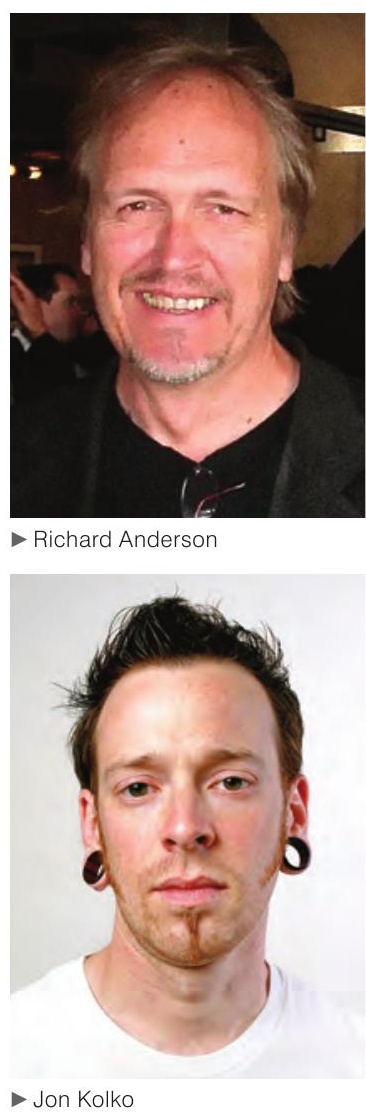

- Jon Kolko

\footnotetext{
DOI: $10.1145 / 1806491.1806492$ (c) 2010 ACM 1072-5220/10/0700 $\$ 10.00$
} 